\title{
Understanding steroidogenesis in polycystic ovary syndrome
}

\section{Estefanía Ventura-Arizmendi and Marcelino Hernández-Valencia*}

Instituto Mexicano del Seguro Social, Centro Médico Nacional Siglo XXI, Specialty Hospital, Endocrine Diseases Medical Research Unit, Ciudad de México, Mexico

\begin{abstract}
Polycystic ovary syndrome is the most common endocrine disease in reproductive age, characterized by menstrual alterations, clinical or biochemical hyperandrogenism, and ultrasound-identified ovarian cysts. The neuroendocrine and metabolic alterations that accompany this condition involve the desensitization of the hypothalamus-pituitary-ovary axis, steroidogenesis and hyperandrogenism; recently, the role of insulin resistance has been explored. Hyperandrogenism has been established to be the main cause of polycystic ovary syndrome, due to enzymatic alterations in the steroidogenic pathway that cause luteinizing hormone over-stimulation because of quick pulses generated by gonadotropin-releasing hormones. Various growth factors of and cytokines inhibit the conversion of androgens into estrogens; activin and prostaglandins are also involved, even high levels of insulin participate in the characteristic deregulation of this syndrome.
\end{abstract}

KEY WORDS: Polycystic ovary syndrome. Steroidogenesis. Hyperandrogenism. Aromatization.

\section{Introduction}

Polycystic ovary syndrome (PCOS) is the most common endocrine disease that affects women of childbearing age. Its prevalence has been reported at between 4 and $8 \%$. $^{1}$ To understand the disease, it is necessary to have an insight into the hypothalamic-pituitary-ovarian axis, ovarian physiology and the pathophysiology of PCOS.

\section{Neuroendocrine axis activity}

With puberty, maturation of the hypothalamic-pituitary-ovary axis begins, as well as gonadotropin-releasing hormone (GnRH) secretion, ${ }^{2}$ which is inhibited during childhood. GnRH release occurs in the form of pulses, on whose frequency and amplitude depends the production of luteinizing $(\mathrm{LH})$ and follicle stimulating (FSH) hormones, ${ }^{3}$ which stimulate ovarian thecal and granulosa cells, respectively. ${ }^{4}$ The former produce androstenedione, while the latter aromatize for the conversion to estradiol. The result is a change in the metabolic pathway towards estrogens, which is expressed with growth of the breasts and bones and gynecoid-type fat deposit. ${ }^{5}$ Other peptides that induce LH receptors and steroidogenic enzymes expression during thecal cell early development have been studied in granulosa cells. ${ }^{6}$

Hence, the production of androgens by the adrenal gland in women is highly important. ${ }^{7}$ During this period, cortical cells, through the adrenocorticotropic hormone at Zona fasciculata, also release large amounts of androgens, such as dehydroepiandrosterone (DHEA) and DHEA sulfate, which are responsible for
Gac Med Mex. 2019;155:169-174

Contents available at PubMed www.gacetamedicademexico.com 
the development of pubic and axillary hair and acne. The increase in ovarian-origin androgens also facilitates the development of pubic hair growth. ${ }^{5}$

This is how two intertwined phenomena coexist: steroidogenesis and folliculogenesis, both dependent on the hypothalamic-pituitary-ovary axis, ${ }^{8}$ which is orchestrated by gonadotropins' neural network, which are released from vesicles of neuron efferent terminals in the middle eminence and the pituitary portal system. FSH secretion promotes LH receptors expression, initial stimulation for follicular development and aromatization in granulosa cells from androstenedione to estradiol. LH is recognized for its participation in the luteal phase as a promoter of progesterone secretion. In addition to its high activity in the follicular phase, it is responsible for inducing the synthesis of androgens and initiating the maturation of the oocyte. Continuous exposure of the pituitary gland by gonadotropins stimulation causes desensitization, and their secretion is thereby suppressed as a feedback pathway.

Recently, GnRH maturation and sensitization activity has been described to depend on a 145-amino acid polypeptide known as kisspeptin. The gene that encodes kisspeptin (KISS1) has been located on chromosome 1. Neurons expressing kisspeptin are present in the arcuate nucleus, the periventricular nucleus and the periventricular anteroventral nucleus in the mouse. Additionally, they are also found in the pre-optic anterodorsal area and the nucleus of the stria terminalis bed. ${ }^{9}$

$\mathrm{GnRH}$ and kisspeptin in the hypothalamus have been assumed to be crucial components of the hypothalamic-pituitary-ovary axis and to maintain the reproductive function by stimulating follicular growth and sex steroids' synthesis. ${ }^{10}$ Kisspeptin signaling in the medial basal hypothalamus has been established to be responsible for the generation of GnRH pulses, ${ }^{11}$ since the absence of kisspeptin induces a state of immaturity of the hypothalamus-gonadal axis, where, by blocking its GPR-54 receptor, the functionality of this pathway is lost, while addition of the kisspeptin polypeptide restores the axis functionality and pulses. ${ }^{12}$

Most neurotransmitters and neuromodulators have excitatory and inhibitory properties depending on certain factors, such as the neurocircuit composition, the state of bodily development throughout life and the hormonal environment. Irregularities in $\mathrm{GnRH}$ release have been established as one of the causes of PCOS origin during puberty. ${ }^{2}$

There is another neuroendocrine regulation mechanism with hypothalamic activity inhibitory or stimulating effect, which acts through neurotransmitters such as gamma-aminobutyric acid (GABA), neuropeptide $Y$ (NPY) and melatonin, which inhibit hypothalamic activity; or glutamate, aspartic acid (N-methyl-d-aspartic acid, NMDA), norepinephrine and glial cells, which have stimulatory activity.13

\section{Ovarian steroidogenesis}

Normal folliculogenesis depends on intra-ovarian androgens for the synthesis of estradiol, as described in the "two gonadotropins-two cells" theory. ${ }^{14}$ This theory about androgen biosynthesis refers that thecal cells secrete androgens in response to $\mathrm{LH}$ and that androstenedione is transformed into estrogens by the action of aromatase in granulosa cells by influence of $\mathrm{FSH}$, which requires for a delicate balance to exist. ${ }^{15}$ Thus, the excess in the production of androgens is a consequence of a disorder in the folliculogenesis process, which is expressed with poor follicular maturation and an increase in follicular atresia. ${ }^{16}$

The initial step in the biosynthesis of all steroid hormones is the conversion of cholesterol into pregnenolone by two pathways that involve the lateral cholesterol anchor chain or the steroidogenesis-modifiable regulatory protein. Subsequently, pregnenolone is converted to dehydroepiandrosterone through two $\Delta 5$ steroid enzyme-dependent steps and the conversion is catalyzed by cytochrome P450c17a. Progesterone undergoes a transformation in parallel to androstenedione via the $\Delta 4$-steroid pathway ( $\Delta 4-5$ isomerase); apparently, these pathways in humans are more dependent on the 17-20 lyase enzyme to arrive to androstenedione by both syntheses, before the activity of 17-ketoreductase to testosterone or estradiol. In the adrenal gland, 17-hydroxyprogesterone is converted into cortisol or sex hormones, depending on whether the 21-hydroxylase pathway is used for cortisol or 17,20 -lyase for 17 -ketosteroids. The action of 17- $\beta$-hydroxydehydrogenase is essential for the conversion of 17-ketosteroids into testosterone, dihydrotestosterone and estradiol (Figure 1)..$^{15} \mathrm{LH}$ acts on the interstitial stroma of ovarian thecal cells, inducing the secretion of androstenedione, and subsequently influences on its conversion into estradiol by the action of aromatase. ${ }^{17}$

The development of the dominant follicle is associated with increased levels of estradiol, which is dominant over androgen concentration; however, it is not a negative feedback mechanism. Thecal cells coordination and granulosa cells function, and the subsequent androgen 


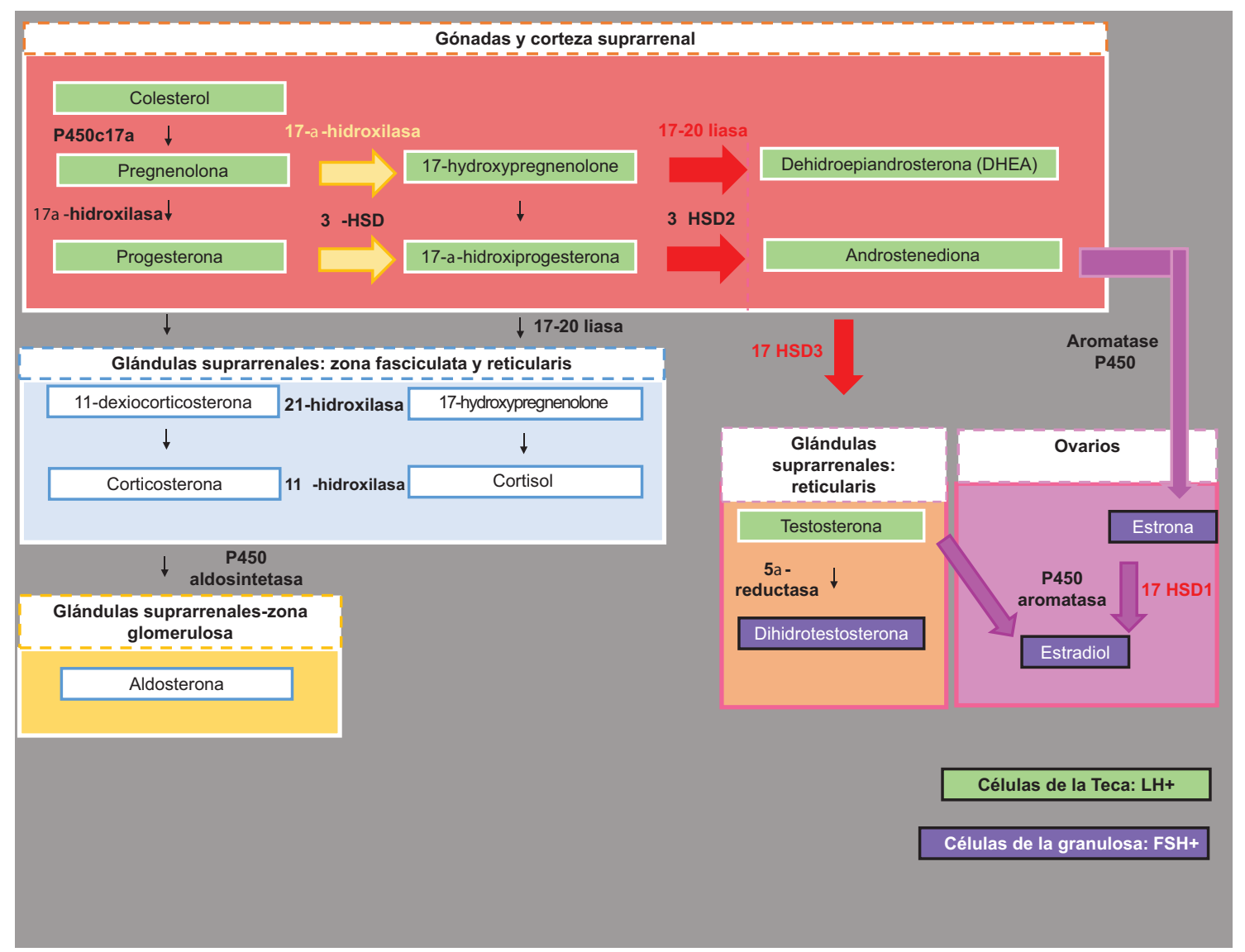

Figure 1. Ovarian and adrenal steroidogenesis. The metabolic pathways in the different glandular compartments are shown. Each compartment complies with the secretion of hormones for this delicate balance. The adrenal glands are divided into cortex and medulla (Zona fasciculata, reticularis and glomerulosa). The red box represents the gonads and the adrenal cortex, where cholesterol, the precursor of this metabolic pathway, is transformed through a series of enzymatic steps into progesterone and testosterone; in turn, at the Zona reticularis, the latter is freely transformed into dihydrotestosterone. The blue box is the Zona fasciculata and reticularis of the adrenal gland where the secretion of glucocorticoids, such as cortisol, is carried out, while the mineralocorticoids (aldosterone) are depicted in the yellow box. Finally androgen aromatization to estradiol occurs by the action of aromatase P450 in the ovary.

synthesis, are finally organized by a combination of autocrine, paracrine and endocrine modulators. Growth factors such as growth and differentiation factor-9 (GDF-9) and bone morphogenetic protein-15 (BMP-15), which act predominantly on granulosa cells through paracrine signaling, participate in the transition from primordial to primary follicle. ${ }^{18}$

\section{Steroidogenesis in PCOS}

Evidence suggests that the onset of PCOS is due to an increase in androgens resulting from steroidogenic dysregulation; ${ }^{19}$ as a consequence of this increase, there is limited estrogenic response. LH time- and dose-dependent over-stimulation, resulting from $\mathrm{LH}$ receptors negative regulation, reduces cholesterol anchoring activity and 17,20 lyase and 17-idroxylase activity, with the subsequent increase in 17-hydroxyprogesterone to androgens. ${ }^{20}$ In patients with PCOS, LH concentration is elevated by 40 to $60 \%$ in comparison with the control population, ${ }^{21}$ due to an increase in the amplitude and frequency of $\mathrm{LH}$ pulses. $^{22}$

The development of granulosa cells, and hence the increase in aromatase activity, also determines the production of androgens. A healthy follicle $\geq 8 \mathrm{~mm}$ in diameter efficiently converts androstenedione to estradiol. In contrast, atretic or cystic follicles have a high proportion of androstenedione conversion to estradiol.

The action of $\mathrm{FSH}$ on granulosa cells determines the growth of healthy follicles to up to $2.5 \mathrm{~mm}$ in diameter, partly mediated by the insulin-like growth factor (IGF) system and insulin physiological concentrations, which together stimulate estradiol production. The IGF-binding protein inhibits FSH bioactivity and is markedly expressed in atretic follicles. Inhibin $B$ is a peptide that is reciprocally regulated by $\mathrm{FSH}$ with a 


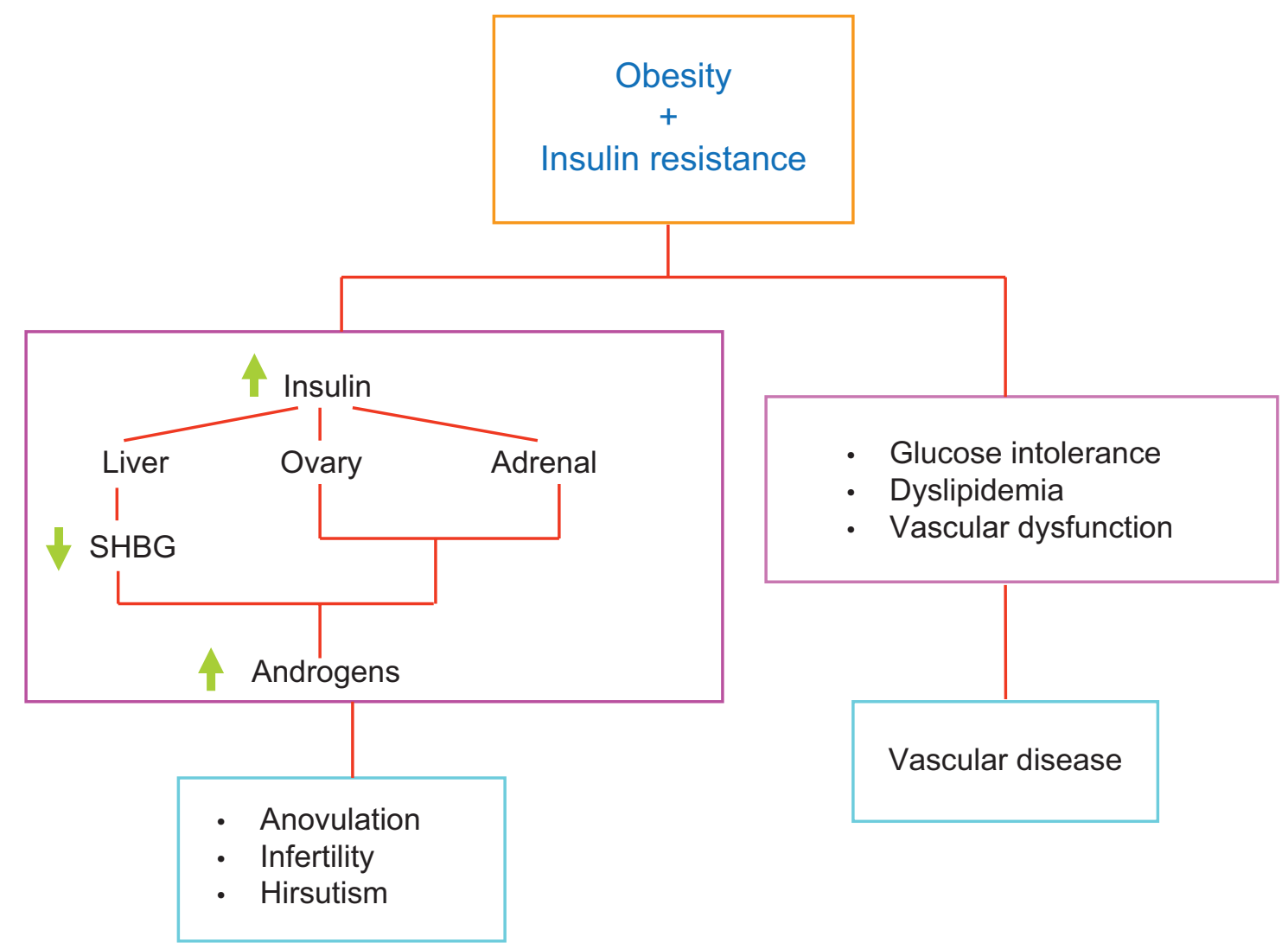

Figure 2. Polycystic ovary syndrome (PCOS) pathophysiology. The hyperinsulinemic state that modifies hepatic, ovarian and adrenal activity with subsequent systemic hormonal alterations that lead to the PCOS phenotypic disorders and infertility, as well as to systemic vascular disorders, are described.

negative feedback, and is essential to promote androgen synthesis from thecal cells, while androgens themselves successively stimulate its production, which is not yet clear because, under normal conditions, FSH predominantly appears to reduce testosterone levels. ${ }^{23}$ In addition, prostaglandins and angiotensin are also promoters, while corticotropin-releasing hormone and $\beta$-growth, epidermal growth and tumor necrosis factors and cytokines have an inhibitory function in androgen biosynthesis. This may be due to the fact that the epidermal and growth factors inhibit aromatase, whereas activin stimulates granulosa cells to produce estrogen, while inhibiting androgen secretion from the theca, and thus, when this regulation is lost, the characteristic hyperandrogenic state of PCOS predominates. $^{15}$

This way, insulin increases LH stimulation in androgenic production. While FSH regulates the following steps in the folliculogenesis process through the selection of dominant follicles, androgens and estrogens are negative modulators of $\mathrm{LH}$ effect, since insulin-like growth factor synergistically has a positive effect on follicular growth. ${ }^{15}$
Androgenic synthesis is also correlated with the development of granulosa cells and is associated with aromatase activity. Efficient conversion of androstenedione to estradiol occurs in healthy follicles $>8 \mathrm{~mm}$ in diameter, and this mechanism is therefore interrupted by atretic and cystic follicles, which results in elevated androstenedione. As a consequence, FSH bioactivity of is inhibited by IGF proteins, which are significantly expressed in atretic follicles. Transforming and fibroblast growth factors also inhibit aromatase and, consequently, estradiol synthesis. ${ }^{15}$

Patients with PCOS have a tendency to estradiol excess in all phases of follicular maturation. PCOS-related cells have been reported to lose response to FSH and to produce less progesterone. ${ }^{24}$

Adrenal hyperandrogenism is a type of androgenic dysfunction characterized by hyper-response to DHEA and hypersensitivity to adrenocorticotropic hormone; however, experimental studies with $\mathrm{GnRH}$ analogues have shown a slight, although significant, decrease in androgens, in contrast to what occurs with dexamethasone, which does not induce changes in androgen 

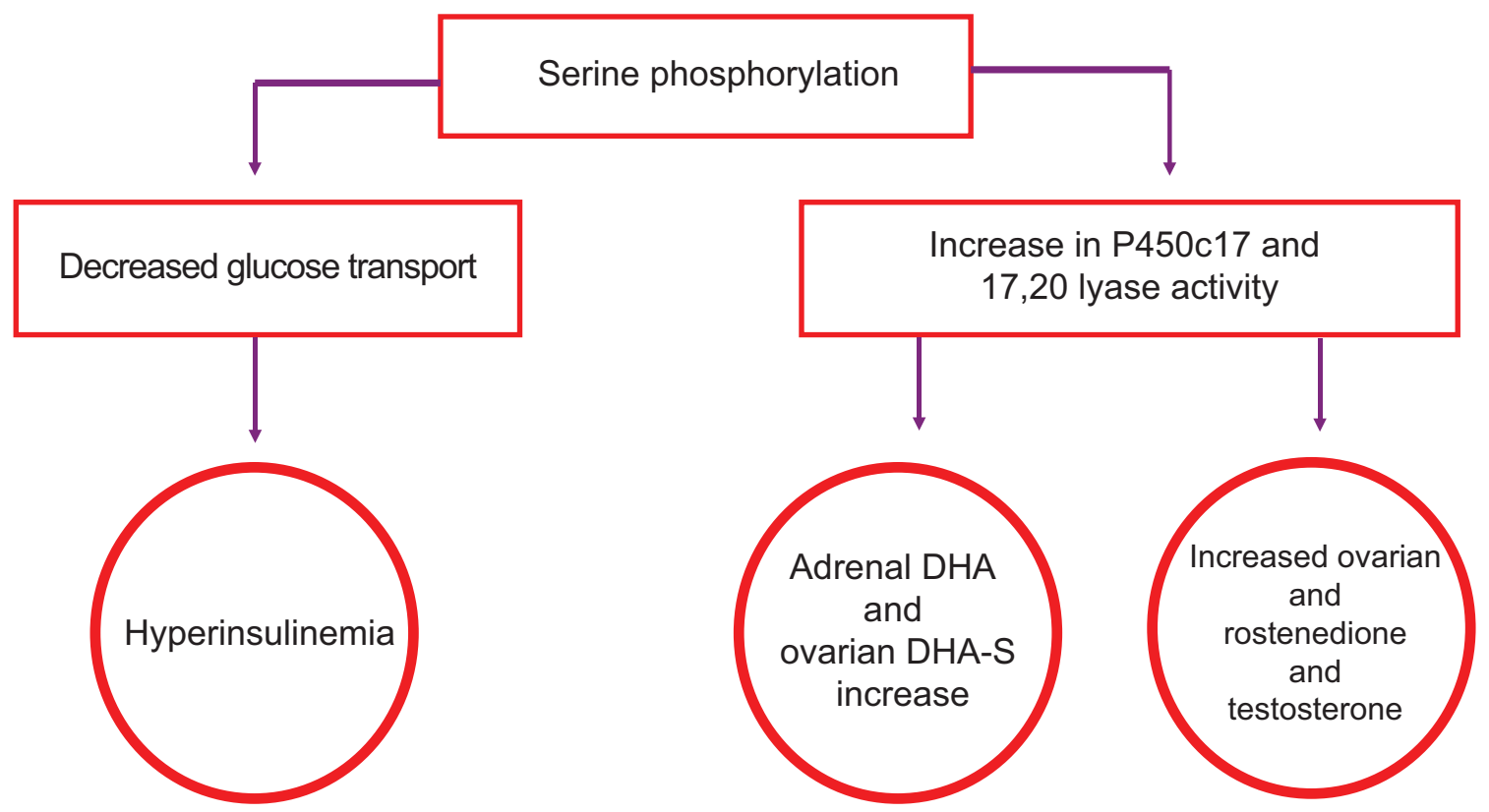

Figure 3. Defects in transduction signals. Subclinical inflammatory states and an altered metabolic state induce serine abnormal phosphorylation in the insulin receptor substrate and, consequently, the blockage of the metabolic and enzymatic pathways that alter the entire steroidogenic state.

concentrations, which in turn suggests that the origin of androgens in PCOS is ovarian, although this event has not been fully clarified. ${ }^{25}$

\section{PCOS pathophysiology}

PCOS pathophysiology is complex because it involves interactions with genetic, metabolic, fetal and environmental factors. Predominant alterations include disorganized gonadotropin secretion, hyperandrogenemia, insulin resistance, hyperinsulinemia, ovarian dysfunction and follicular interruption (Figure 2). ${ }^{26} \mathrm{~A}$ constant characteristic of PCOS is an increase in LH levels, decreased or normal FSH values and persistently rapid pulse frequency in GnRH secretion. ${ }^{27}$ The role of hyperandrogenism in $\mathrm{GnRH}$ steroidal regulation and gonadotropin secretion is uncoordinated. LH hypersecretion affects ovarian androgen synthesis, folliculogenesis and oocyte development. ${ }^{28}$

CYP17A1 (cytochrome P450, family 17 , subfamily A, member 1 ) is a monooxygenase that is located in the endoplasmic reticulum, which catalyzes numerous reactions that involve the metabolism of various drugs, as well as the synthesis of cholesterol, steroids and other lipids through at least 10 enzymes that are found in the mitochondria of different tissues with steroidogenic capacity such as the ovaries, testes, adrenal glands, placenta, adipocytes and some areas of the brain. This cytochrome catalyzes 17-hydroxylase and the subsequent 17,20 lyase reactions with pregnenolone, progesterone and allopregnanolone. Therefore, it is an enzymatic key of the steroidogenic pathway for the production of progestins, mineralocorticoids, glucocorticoids, androgens and estrogens. Mutations of this gene are associated with 17- $\alpha$-hydroxylase and 17-0-hydroxylase/17,20-lyase steroids deficiency, which causes pseudohermaphroditism and congenital adrenal hyperplasia. ${ }^{29,30}$

Several studies indicate that hyperandrogenemia in anovulation and ovulation conditions in women with PCOS originates predominantly in the ovary; in addition, they confirm that the primary cause of excessive androgen production in PCOS is not only related to $\mathrm{LH}$ hypersecretion and, therefore, it is concluded that the intrinsic defect is related to thecal cell dysfunction in the ovary or other stimulatory influences, such as insulin and IGF-1, among other cytokines. ${ }^{31}$

$\mathrm{LH}$ has been described to act on granulosa cells in the presence of insulin and to lead to early luteinization, follicular development interruption and excessive androgen production. ${ }^{24}$

In the development of obesity, insulin resistance is also related to tumor necrosis factor alpha, since it increases serine phosphorylation ${ }^{32}$ (Figure 3) in the insulin receptor signaling pathway and its substrates, thus inhibiting its signaling, and modifying ovarian functioning. ${ }^{?}$ 


\section{Final considerations}

PCOS is the most common endocrine disease that affects women of childbearing age. Existing information indicates that it is caused by a deviation in the steroidogenesis pathway, with a consequent increase in androgen levels resulting from hormonal deregulation in the ovary. Thus, growth and epidermal factors in the ovary inhibit aromatase, whereas activin promotes the production of estrogen by granulosa cells, while inhibiting thecal androgen secretion, which is why PCOS characteristic hyperandrogenic state predominates when this regulation is lost.

\section{References}

1. Sirmans SM, Pate KA. Epidemiology, diagnosis, and management of polycystic ovary syndrome. Clin Epidemiol. 2014;6:1-13.

2. Terasawa E, Fernandez DL. Neurobiological mechanisms of the onset of puberty in primates. Endocr Rev. 2001;1:111-151.

3. Besser GM, McNeilly AS, Anderson DC, Marshall JC, Harsoulis P Hall $R$, et al. Hormonal responses to synthetic luteinizing hormone and follicle stimulating hormone-releasing hormone in man. Br Med J. 1972; 3:267-271.

4. Ciccone NA, Kaiser UB. The biology of gonadotroph regulation. Cur Opin Endocrinol Diabetes Obes. 2009:16:321-327

5. Roe AH, Dokras A. The diagnosis of polycystic ovary syndrome in adolescents. Rev Obstet Gynecol. 2011;4:45-51

6. Liu C, Peng J, Matzuk MM, Yao HH. Lineage specification of ovarian theca cells requires multicellular interactions via oocyte and granulosa cells. Nat Commun. 2015;6:6934-6955.

7. Baptiste CG, Battista MC, Trottier A, Baillargeon JP. Insulin and hyperandrogenism in women with polycystic ovary syndrome. J Steroid Biochem Mol Biol. 2010;122:42-52.

8. Baskind NE, Balen AH. Hypothalamic-pituitary, ovarian and adrenal contributions to polycystic ovary syndrome. Best Pract Res Clin Obstet Gynaecol. 2016;37:80-97.

9. Gianetti E, Seminara S. Kisspeptin and KISS1R: a critical pathway in the reproductive system. Reproduction. 2008;136:295-301.

10. Oride A, Kanasaki H, Mijiddorj T, Sukhbaatar U, Ishihara T, Kyo S. Regulation of kisspeptin and gonadotropin releasing hormone expression in rat placenta: study using primary cultures of rat placental cells. Reprod Biol Endocrinol 2015;13:90-98.

11. Ezzat A, Pereira A, Clarke IJ. Kisspeptin is a component of the pulse generator for $\mathrm{GnRH}$ secretion in female sheep but not the pulse generator. Endocrinology. 2015;156:1828-1837.

12. Hanchate NK, Parkash J, Bellefontaine N, Mazur D, Colledge WH, D'Anglemont-De-Tassigny $\mathrm{X}$, et al. Kisspeptin-GPR54 signaling in mouse NO-synthesizing neurons participates in the hypothalamic control of ovulation. J Neurosci. 2012;32:932-945.

13. Donoso AO, López FJ, Negro-Vilar A. Cross-talk between excitatory and inhibitory amino acids in the regulation of luteinizing hormone-releasing hormone secretion. Endocrinology. 1992;131:1559-1561.
14. Gougeon A. Regulation of ovarian follicular development in primates: facts and hypotheses. Endocr Rev. 1996;17:121-155.

15. Balen AH, Conway GS, Homburg R, Legro RS. Polycystic ovary syndrome. A guide to clinical management. EE. UU.: Taylor \& Francis; 2005.

16. Rosenfield RL, Ehrmann DA. The pathogenesis of polycystic ovary syndrome (PCOS): the hypothesis of PCOS as functional ovarian hyperandrogenism revisited. Endocr Rev. 2016;37:467-520.

17. Barbieri RL. The endocrinology of the menstrual cycle. En: Rosenwaks Z, Wassarman PM, editores. Human Fertility. Methods and Protocols. EE. UU.: Humana Press; 2014

18. Otsuka F, McTavish K, Shimasaki S. Integral role of GDF-9 and BMP-15 in ovarian function. Mol Reprod Dev. 2011;78:9-21.

19. Rosenfield RL, Barnes RB, Cara JF, Lucky AW. Dysregulation of cytochrome P450c17 alpha as the cause of polycystic ovarian syndrome. Fertil Steril. 1990;53:785-791.

20. White D, Leigh A, Wilson C, Donaldson A, Franks S. Gonadotrophin and gonadal steroid response to a single dose of a long-acting agonist of gonadotrophin-releasing hormone in ovulatory and anovulatory women with polycystic ovary syndrome. Clin Endocrinol (Oxf). 1995;42:475-481.

21. Taylor AE, McCourt B, Martin K, Anderson EJ, Adams J, Schoebfeld D, et al. Determinants of abnormal gonadotropin secretion in clinically defined women with polycystic ovary syndrome. J Clin Endocrinol Metab. 1997:82:2248-2256.

22. Barontini M, García-Rudaz M, Veldhius JD. Mechanisms of hypothalamic-pituitary-gonadal disruption in polycystic ovarian syndrome. Arch Med Res. 2001:32:544-552.

23. Hirshfeld-Cytron J, Barnes RB, Ehrmann DA, Caruso A, Mortensen MM, Rosenfield RL. Characterization of functionally typical and atypical types of polycystic ovary syndrome. J Clin Endocrinol Metab. 2009;94:1587-1594.

24. Mason HD, Willis DS, Beard RW, Winston RM, Margara R, Frank S. Estradiol production by granulosa cells of normal and polycystic ovaries: relationship to menstrual cycle history and to concentrations of gonadotrophins and sex steroids in follicular fluid. J Clin Endocrinol Metab. 1994;79:1355-1360

25. Rosenfield RL, Mortensen M, Wroblewski K, Littlejohn E, Ehrmann DA Determination of the source of androgen excess in functionally atypical polycystic ovary syndrome by a short dexamethasone androgensuppression test and a low-dose ACTH test. Hum Reprod. 2011;26: 3138-3146.

26. Dumesic DA, Oberfield SE, Stener-Victorin E, Marshall JC, Laven JS, Legro RS. Scientific statement on the diagnostic criteria, epidemiology, pathophysiology, and molecular genetics of polycystic ovary syndrome. Endocr Rev. 2015,36:487-525.

27. Kazer RR, Kessel B, Yen SS. Circulating luteinizing hormone pulse frequency in women with polycystic ovary syndrome. J Clin Endocrinol Metab. 1987;65:233-236

28. Qiao J, Feng HL. Extra-and intra-ovarian factors in polycystic ovary syndrome: impact on oocyte maturation and embryo developmental competence. Hum Reprod Update. 2011;17:17-33.

29. RefSeq: NCBI Reference Sequence Database [Sitio web]. EEUU: National Center for Biothechnology Information/National Library of Medicine; 2008.

30. Peng HM, Im SC, Pearl NM, Turcu AF, Rege J, Waskell L, et al. Cytochrome b5 activates the 17,20-lyase activity of human cytochrome P450 17A1 by increasing the coupling of NADPH consumption to androgen production. Biochemistry. 2016:55:4356-4365

31. Balen, A. The pathophysiology of polycystic ovary syndrome: trying to understand PCOS and its endocrinology. Best Pract Res Clin Obstet Gynaecol. 2004:18:685-706.

32. Dunaif A. Insulin resistance and the polycystic ovary syndrome: mechanisms and implication for pathogenesis. Endocr Rev. 1997;18:774-800. 